\title{
Wetland Classification in Poyang Lake Using Dual-polarization Synthetic Aperture Radar Data with Feature Combination
}

\author{
Guanhua Zhou, ${ }^{1}$ Zhiyuan Wang, ${ }^{1}$ Haoyu Miao, ${ }^{1}$ Cheng Jiang, ${ }^{2}$ and Guifei Jing ${ }^{3 *}$ \\ ${ }^{1}$ School of Instrumentation and Optoelectronic Engineering, Beihang University, Beijing 100191, China \\ ${ }^{2}$ Beijing Institute of Space Mechanics \& Electricity, Beijing Key Laboratory \\ of Advanced Optical Remote Sensing Technology, Beijing 100094, China \\ ${ }^{3}$ Research Institute of Frontier Science, Beihang University, Beijing 100191, China
}

(Received August 17, 2021; accepted November 16, 2021)

Keywords: synthetic aperture radar, Sentinel-1, polarimetric decomposition, Poyang Lake, classification

The dual-polarized Sentinel-1 synthetic aperture radar (SAR), which performs C-band SAR imaging day and night regardless of the weather, offers new opportunities for wetland cover monitoring in regions frequently covered by cloud. In this study, a decision tree (DT) classifier was applied to investigate the utility of Sentinel-1 for wetland classification in Poyang Lake, China. Six land cover classes were identified: water body, bare land, aquatic vegetation, cropland, forest, and urban area surrounding the lake. Two types of features were extracted from the dual-polarized SAR data, namely, the backscattering coefficients and the polarimetric decomposition components. Then, the DT classifier was trained and applied with backscattering features, polarimetric features, or a combination of the two features. The overall accuracy of all the classifiers was over $90 \%$ for the different feature combinations $(98.02,90.63$, and $98.59 \%)$ for the classes in the lake area, compared with less than $78 \%(74.84,63.01$, and $77.29 \%)$ when the classes surrounding the lake were also considered, which demonstrates the potential of Sentinel-1 SAR data for wetland monitoring. The accuracy of different feature combinations increased in the following order: polarimetric features $<$ backscattering features $<$ combination of polarimetric and backscattering features. The artificial neural network, naïve Bayes, random forest, and adaptive boost algorithms were compared for the case of using backscattering features, and the adaptive boost algorithm showed lower performance than the other three algorithms.

\section{Introduction}

The Poyang Lake wetland is one of the most important wetlands in the world, providing many ecological services, including purifying water, controlling natural hazards, and conserving soil and water, as well as numerous benefits for human society such as economic and scientific benefits. ${ }^{(1,2)}$ Remote sensing techniques, mainly using optical, IR, and radar sensors, are widely used to map and monitor wetlands. However, because optical and IR remote sensing are strongly affected by cloud and rain, it is difficult to obtain high-quality images of the study area in such weather, limiting their effectiveness and operational feasibility for temporal wetland 
monitoring. ${ }^{(3)}$ On the other hand, owing to its multiband, multipolarization, and multiangle features, synthetic aperture radar (SAR), which can operate continuously under all weather conditions and penetrate the vegetation canopy to some extent, can effectively compensate for the shortcomings of optical and IR remote sensing sensors and is suitable for wetland monitoring. ${ }^{(4)}$

With the launches of the ENVISAT, ALOS PolSAR, RADARSAT-2, TerraSAR, Sentinel-1, and GF-3 satellites, radar remote sensing has been commonly applied in wetland classification and information extraction. Numerous studies based on different bands, polarizations, and classification methods have been conducted by the wetland community. Yamagata and Yasuoka conducted a wetland vegetation classification study using C-band ERS-1 SAR (VV polarization) and L-band JERS-1 SAR (HH polarization) data with symbiotic matrix texture analysis. ${ }^{(5)}$ Rio and Lozano-Garcia used RADARSAT-1 data processed by spatial filtering to study the classification of marsh and wetland, and achieved good precision, indicating the advantages of SAR in wetland classification. ${ }^{(6)}$ Arzandeh and Wang applied gray-level co-occurrence matrix texture analysis to wetland classification using single-date RADARSAT images, and the results showed that this method can effectively improve the classification accuracy of wetlands. ${ }^{(7)} \mathrm{Li}$ and Chen proposed a rule-based wetland classification method using Landsat-7, RADARSAT-1, and digital elevation model (DEM) data, and the results showed that the confusion method of optical remote sensing and SAR was superior to the classification method using optical remote sensing alone or SAR alone. ${ }^{(8)}$ A neural network combined with the Michigan Microwave Canopy Scattering (MIMICS) model, which was modified to fit to herbaceous wetland ecosystems, was used to retrieve information on wetland vegetation biomass in Poyang Lake with ENVISAT ASAR data. ${ }^{(9)}$ Whitcomb et al. used two seasons of L-band SAR imagery to produce a thematic map of wetlands throughout Alaska using the random forests decision tree (DT) algorithm and distinguished as many as nine different classes. ${ }^{(10)}$ Full-polarization SAR systems are able to extract expanded information of the targets; thus, recent studies have mainly focused on the applications of full-polarization SAR. Lönnqvist et al. compared four methods of wetland classification using ALOS PolSAR full-polarization data, where two methods were based on supervised classification and two methods were based on unsupervised classification, and the intensity data were also used. ${ }^{(11)}$ The results showed that the classification result of the full-polarization data was better than that of the intensity data regardless of the method used. Different polarization decomposition methods have been gradually applied to wetland classification using full-polarization SAR data, and high classification accuracy has been achieved by combining different classifiers. Touzi proposed a new coherent target scattering vector model, which was applied to wetland classification and achieved good results. ${ }^{(12)}$ Patel et al. used L-band and P-band full-polarization DLR-ESAR data to decompose a wetland target based on the feature vector, demonstrating the ability of PolSAR technology to characterize the different scattering performances of each component of a wetland ecosystem. ${ }^{(13)}$ Touzi proposed that the target scattering type can be represented by the symmetrical scattering type, and used the amplitude and phase for wetland classification, enabling the $\mathrm{HH}$ and VV phase difference and radiation scattering information to be distinguished; then, they used Touzi decomposition to classify wetlands and provide novel information. ${ }^{(14)}$ Liao and Wang used the 
$H-A-\alpha$ polarimetric decomposition method to extract the polarimetric features of the different land types of cover in Poyang Lake wetland by using RADARSAT-2 data. ${ }^{(15)}$ Unsupervised and supervised Wishart classification were conducted and both obtained high accuracy, suggesting that the RADARSAT-2 data can not only ensure high recognition ability, but can also be used to distinguish between different types of land cover of wetland. Chen et al. extracted polarization parameters by the Cloude-Pottier decomposition method using full-polarization PolSAR data, and then classified coastal wetlands in Jiangsu Province (China) by combining hierarchical clustering and the fuzzy c-means algorithm. ${ }^{(16)}$ The overall classification accuracy reached $86.93 \%$, effectively demonstrating the application potential of L-band full-polarization data in coastal wetland classification.

However, compared with the dual-polarization SAR systems, full-polarization SAR systems have a narrower swath width, larger data volume, lower resolution, and higher antenna transmitter power requirements, which limit the availability of data and hence restrict their applications in large-scale wetland research. Instead, dual-polarization SAR systems with wide coverage and a higher signal-to-noise ratio are mounted on most satellites in orbit and about to be launched. Moreover, the focus of research has gradually shifted to the combined application of dual-polarization SAR, and results show that its classification accuracy is close to that of fullpolarization SAR. ${ }^{(17)}$ Therefore, at the expense of losing some scattering properties of the target, dual-polarization SAR systems are commonly used. ${ }^{(18,19)}$ Moreover, Sentinel-1 provides a large amount of free dual-polarization SAR data for remote sensing applications on wetland. Adeli et al. conducted a comprehensive review of wetland monitoring using SAR data based on a meta-analysis and highlighted the current challenges in this community and their solutions. One of the challenges is the similar backscattering of different wetland classes. Choosing the appropriate SAR specifications (such as incident angle, band, and polarization) according to the wetland type and phenological stage is also a subject of debate and should be investigated more thoroughly. ${ }^{(20)}$ Therefore, the aim of this study is to investigate the application potential of Sentinel-1 for Poyang Lake wetland classification by the DT method using three different feature combinations to explore whether an integrated feature provides improved classification accuracy and to identify the optimum features for wetland classification.

\section{Materials and Methods}

\subsection{Study area}

As the largest freshwater lake wetland in China, Poyang Lake has all the characteristics of a wetland in terms of its spatial and temporal distributions and ecological characteristics. The study area is located in the north of Jiangxi Province from latitudes $28^{\circ} 22^{\prime}$ to $29^{\circ} 45^{\prime} \mathrm{N}$ and longitudes $115^{\circ} 47^{\prime}$ to $116^{\circ} 45^{\prime}$ E (Fig. 1). Poyang Lake is an ephemeral lake, with large differences in the cover area and water storage between the rainy and dry seasons. During the rainy season, the water level rises sharply and the area of the lake can reach $3500 \mathrm{~km}^{2}$. In contrast, during the dry season, the lake may shrink to less than $1000 \mathrm{~km}^{2}$ and become a system of sub-lakes interspersed with mudflats, sediment beds, and vegetation. 


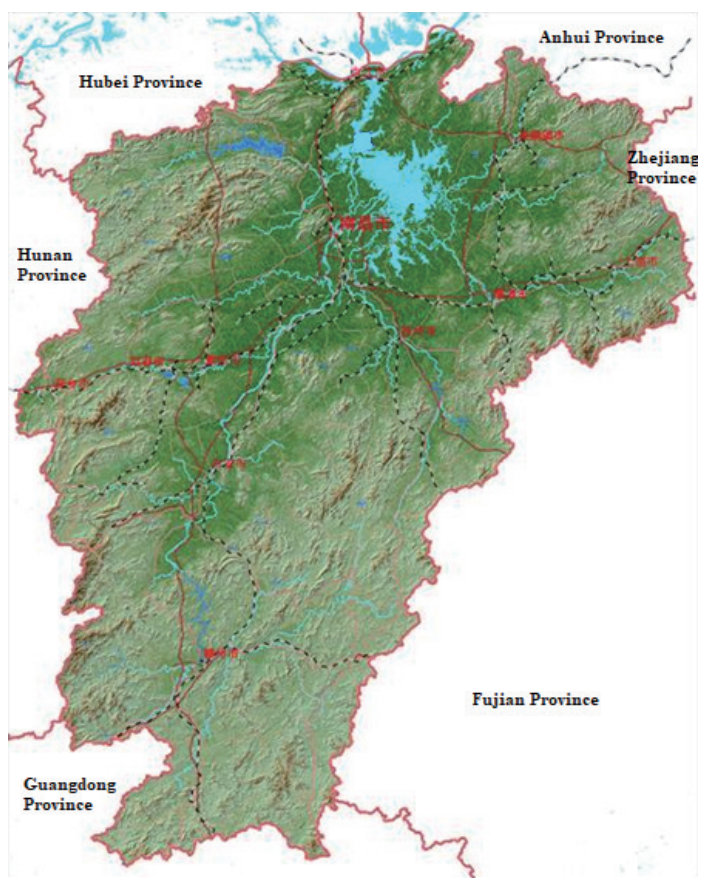

(a)

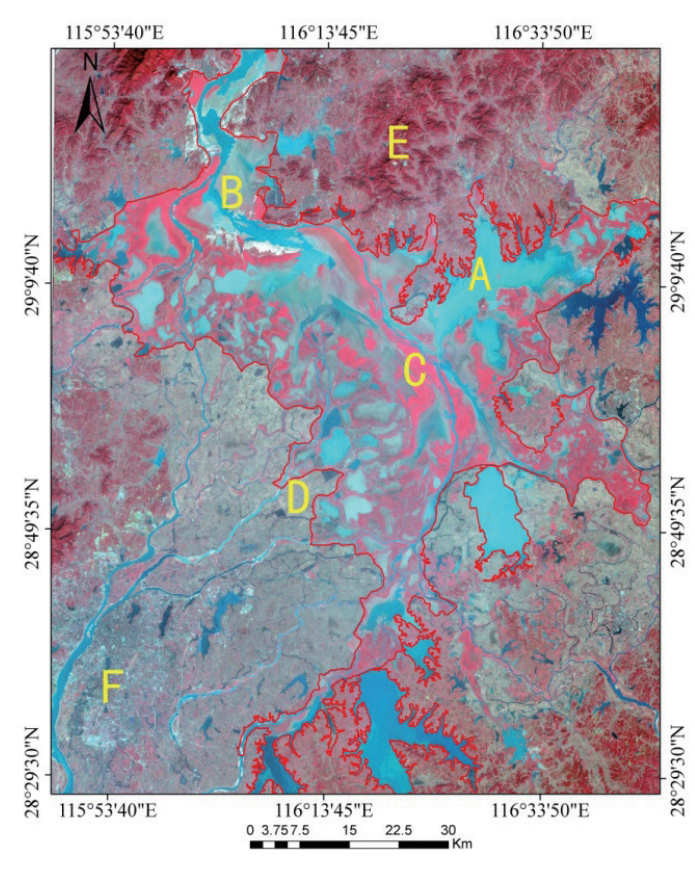

(b)

Fig. 1. (Color online) Location and extent of the study area of Poyang Lake, Jiangxi Province, China. (a) Location map and (b) Landsat-8 OIL false-color composite acquired on December 16, 2016 (R: NIR; G: red; B: green).

Figure 1 shows the location and extent of the study area. The Landsat- 8 OIL false-color composite shows the extent of the image acquired on December 16, 2016, and the red polygon shows the extent of the Poyang Lake wetland. Six land cover classes can be identified from the image: water body, bare land, aquatic vegetation, cropland, forest, and urban area. The water body (region A) appears blue in the image, but the color tone varies with the water quality and sediment content. Bare land (region B) mostly appears brown and white. Aquatic vegetation (region $\mathrm{C}$ ) is mainly red due to a reflection peak in the near-IR band. Beyond the red boundary line, the main types of land surface are cropland (region D), forest (region E), and urban area (region F). Because there is less vegetation in winter, the cropland surface is brown bare soil, mainly located on the east and west sides of Poyang Lake. Because of the vegetation coverage, the forest is red and is mainly located in the upper left, upper right, and lower right corners of the image. The urban area is mainly located in the lower left corner of the image, covering the area of Nanchang City and appearing as a dark color.

\subsection{Sentinel-1 data}

Sentinel-1 is a two-satellite constellation designed to deliver a wealth of data and imagery that are central to Europe's ambitious Copernicus program. Sentinel-1, first launched in 2014, provides users with free C-band images available in single- and dual-polarized modes. ${ }^{(21)}$ As an advanced radar mission, Sentinel-1 can image the surface of Earth through cloud and rain regardless of whether it is day or night. This makes it ideal for monitoring wetlands, which are 
typically shrouded by cloud during the rainy season. The SAR images used in this paper were downloaded from the Copernicus Open Access Hub (https://scihub.copernicus.eu/dhus/\#/home).

\subsection{Sentinel-1 data processing}

Sentinel-1 SAR provides Single Look Complex (SLC) data and Ground Range Detected (GRD) data for downloading, which were simultaneously downloaded in this study. Level-1 GRD products consist of focused SAR data that have been detected, multi-looked, and projected to ground range using an Earth ellipsoid model. Note that phase information is lost. The resulting product has approximately square resolution pixels and square pixel spacing with reduced speckle at the cost of reduced geometric resolution. In contrast, level-1 SLC products consist of focused SAR data geo-referenced using orbit and attitude data from the satellite and provided in a zero-Doppler slant-range geometry. The products include a single look in each dimension using the full TX signal bandwidth and consist of complex samples that preserve the phase information. Therefore, GRD data were used to extract radar backscattering coefficients whereas SLC data were used to extract the polarimetric decomposition parameters. The data preprocessing was mainly carried out by Sentinel Application Platform (SNAP) software provided by the European Space Agency (ESA). First, the two types of level-1 images were split, debursted, mosaicked, and then subsetted to obtain the desired area of interest. It was necessary to limit the processing time of large scenes. Next, for the GRD data, the processing steps included radiometric calibration, speckle filtering, and geometric correction; then, the backscattering coefficients $\sigma_{\mathrm{VV}}^{0}, \sigma_{\mathrm{VH}}^{0}$, and $\sigma_{\mathrm{VH}^{-}}^{0} \sigma_{\mathrm{VV}}^{0}$ were calculated. Compared with GRD data, SLC data processing was more complex and consisted of radiometric calibration, multilook processing, polarimetric matrix generation, polarimetric speckle filtering, and polarimetric decomposition. A refined Lee filter with a window size of $7 \times 7$ was used to reduce the speckle. After that, the polarimetric components (i.e., entropy, alpha, and anisotropy) were extracted from $H-\alpha$ dual-polarization decomposition. ${ }^{(22)}$ Finally, all the extracted data were projected to the same projection, which could be easily used to extract the same backscattering coefficients and the polarimetric decomposition components of the sample points from their coordinates.

\subsection{Classification and accuracy assessment}

A DT is a non-parametric supervised learning method that has been used successfully for a wide range of classification problems. The goal of the method is to create a model that predicts the value of a target variable by learning simple decision rules inferred from the data features. It is simple to understand and interpret, can handle both numerical and categorical data, and performs well with large datasets. In this paper, the DT method was implemented using Python's scikit-learn library. ${ }^{(23)}$

Figure 1 shows that the water body, bare land, and aquatic vegetation are the main land cover classes in the Poyang Lake region, and there are three other land cover classes around the lake: cropland, forest, and urban area. Therefore, classification was conducted in two cases, with one case including only three land cover classes and the other case including six land cover classes. 
Table 1

Feature combinations for land cover classification.

\begin{tabular}{lc}
\hline Group & Combination of features \\
\hline 1 & $\sigma_{\mathrm{VV}}^{0}, \sigma^{0}{ }_{\mathrm{VH}}, \sigma^{0}{ }_{\mathrm{VH}}-\sigma_{\mathrm{VV}}^{0}$ \\
\hline 2 & entropy, alpha, anisotropy \\
\hline 3 & $\sigma_{\mathrm{VV}}^{0}, \sigma_{\mathrm{VH}}^{0}, \sigma^{0}{ }_{\mathrm{VH}}-\sigma_{\mathrm{VV}}^{0}$, entropy, alpha, anisotropy \\
\hline
\end{tabular}

Firstly, samples of three feature combinations were extracted from the images according to Table 1, including 224297 water body samples, 103534 bare land samples, 102825 aquatic vegetation samples, 123364 cropland samples, 125188 forest samples, and 108127 urban area samples. Secondly, samples were randomly segmented, with $75 \%$ of the samples used for training and $25 \%$ of the samples used for testing. Then, DTs were trained using the training sets. In the training process, the optimal values of the main parameters of the DT, such as the depth of the DT and the index of feature selection, were automatically determined by the grid search method (which is used to traverse a variety of parameter combinations systematically and determine the most effective parameters through cross-validation). Finally, the classification accuracy was tested by the test set, and a confusion matrix was constructed to evaluate the accuracy. To obtain the training and validation sample data used for classification, a highresolution Google Earth map was used to obtain simple polygons for training and validation, which could be overlaid on the extracted images using coordinates. In addition, four machine learning algorithms based on the optimal feature combination were tested.

\section{Results and Discussion}

\subsection{Feature analysis of land cover classes}

\subsubsection{Backscattering analysis}

Figure 2 shows a false-color composite image of the study area obtained using backscattering coefficients $\sigma_{\mathrm{VV}}^{0}, \sigma_{\mathrm{VH}}^{0}$, and $\sigma_{\mathrm{VH}}^{0}-\sigma_{\mathrm{VV}}^{0}$. The water body appears blue, mainly because the water surface is smooth and prone to specular reflection, which leads to low backscattering values in both polarizations. Aquatic vegetation has a color between blue and white, mainly because the $\mathrm{VH}$ polarization is greatly affected by vegetation coverage. Bare land is mainly brown as the scattering mechanism of exposed soil is surface scattering. For the urban area, dihedral reflection occurring between buildings increases the backscattering values in both polarizations, producing a yellow color. Forest appears bright white because of the strong backscattering from the canopy. Cropland, which is lightly vegetated, has a color between brown and white.

A backscattering separability analysis was performed by examining the distribution of the pixel backscattering coefficients for each class via box-and-whisker plots (Fig. 3) to gain a broad understanding of the radiometric characteristics of each thematic class, as well as the pixel value variability found within each class. ${ }^{(24)}$ The polarization is useful for discriminating different wetland classes. ${ }^{(25)}$ Figure 3 shows that the water body is most easily distinguished from other classes, especially in VV polarization; aquatic vegetation and bare land can be distinguished 


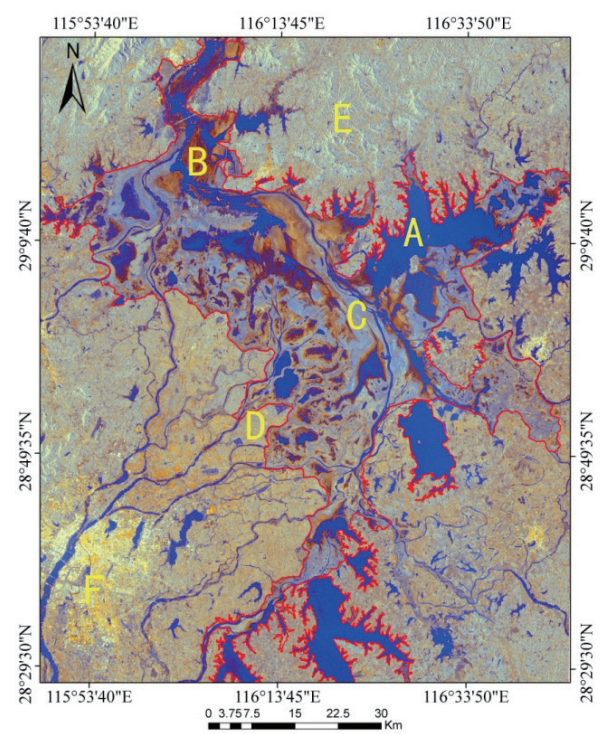

Fig. 2. (Color online) Composite image of Sentinel-1 (R: $\left.\sigma_{\mathrm{VV}}^{0}, \mathrm{G}: \sigma_{\mathrm{VH}}^{0}, \mathrm{~B}: \sigma^{0}{ }_{\mathrm{VH}}-\sigma_{\mathrm{VV}}^{0}\right)$.

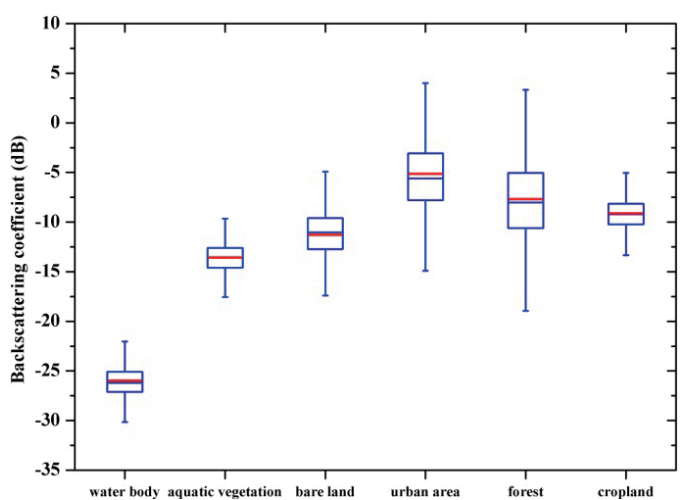

(a)

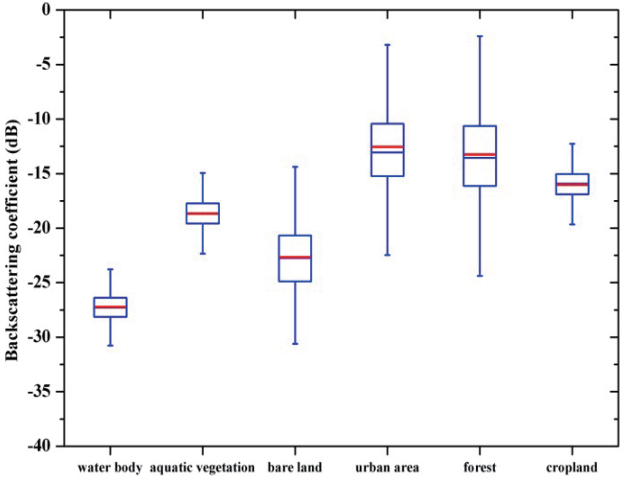

(b)

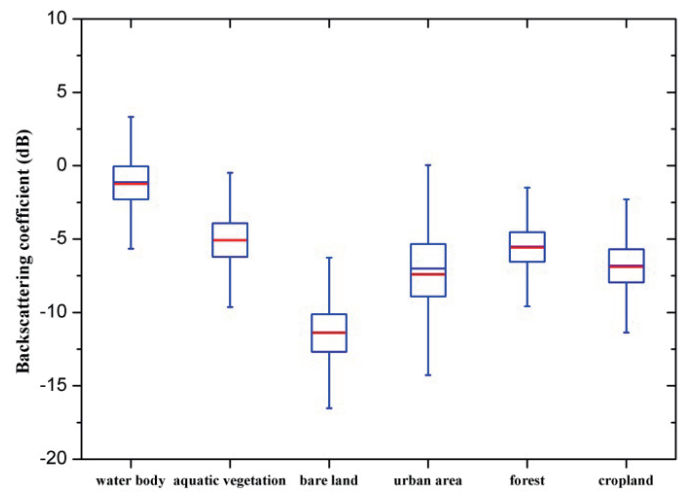

(c)

Fig. 3. (Color online) Box-and-whisker diagrams showing distribution of backscattering coefficients for six classes derived from pixel values extracted from training points. Red bars indicate means, boxes represent the 25th to 75th percentile, and whiskers extend to the minimum and maximum values (excluding outliers). (a) $\sigma_{\mathrm{VV}}^{0}$, (b) $\sigma_{\mathrm{VH}}^{0}$, and (c) $\sigma_{\mathrm{VH}}^{0} \sigma^{0} \mathrm{VV}$. 
using the polarization difference. It is predicted that the three classes of water body, bare land, and aquatic vegetation can be well classified using backscattering coefficients. However, there is a large overlap among the backscattering features for the other three classes, namely, urban area, forest, and cropland, indicating low separability.

\subsubsection{Polarimetric analysis}

Entropy $(H)$, anisotropy $(A)$, and alpha $(\alpha)$ images were obtained for the study area (Fig. 4). Entropy defines the degree of statistical disorder of each distinct scatter type within the ensemble and varies from 0 to 1 . A value of 0 indicates that all the scattering originates from one mechanism, whereas a value of 1 represents a completely random scattering mechanism. ${ }^{(22)}$ In Fig. 4(a), the highest values of $H$ of almost 1 appear in the water body, which is due to the randomness caused by the water surface. For smooth water surfaces, with little or no variation at the scale of the radar wavelength, there is little return energy as the incident wave undergoes specular reflection. Forest and aquatic vegetation show high $H$ values because canopy scattering and dihedral scattering lead to a more complex scattering mechanism. The lowest values of $H$ of close to 0 appear in bare land and urban area, which is due to surface scattering and double bounce scattering, respectively.

Anisotropy, on the other hand, is a measure of the homogeneity of a target relative to the radar look direction. The value of $A$ also varies from 0 to 1 , becoming 0 if both scattering mechanisms are of equal importance. A larger value of $A$ indicates more anisotropic scattering. Thus, anisotropy is complementary to entropy. However, it can be used as a means of decomposition only when $H>0.7$. In Fig. 4(b), the value of $A$ for the water body is close to 0 , while $A$ is close to 1 for cropland and urban area. For forest and aquatic vegetation, $A$ is about 0.4 , making them almost indistinguishable from each other.

Angle $\alpha$ indicates the nature of the dominant scattering mechanism for a resolution cell if $H$ is 0 for the cell. The value of $\alpha$ identifies whether the scattering is volume, surface, or double

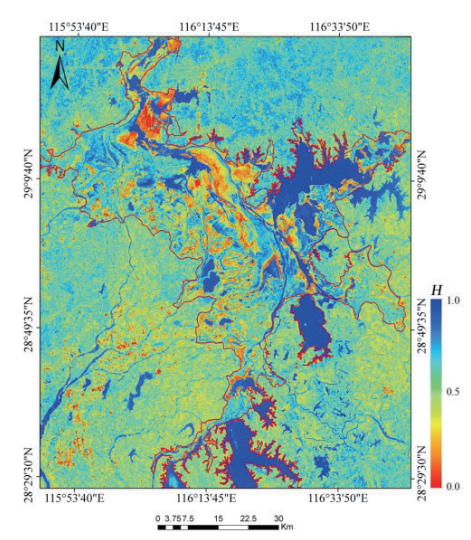

(a)

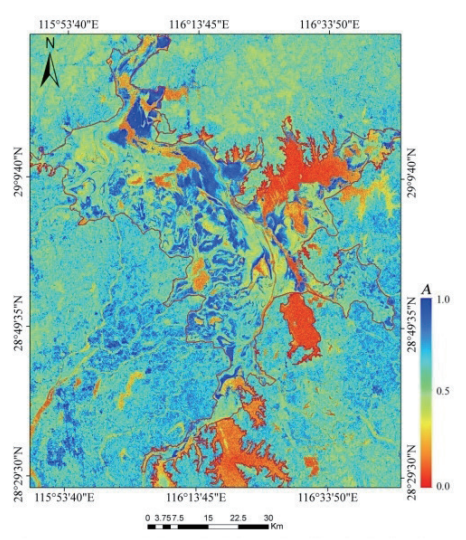

(b)

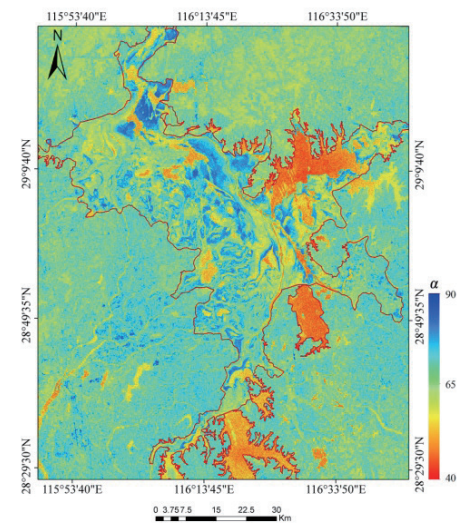

(c)

Fig. 4. (Color online) Images of polarimetric components extracted from study area. (a) Entropy, (b) anisotropy, and (c) alpha. 
bounce. The value of $\alpha$ is normalized between $0^{\circ}$ and $90^{\circ}$, with $\alpha$ close to zero indicating surface scattering, $45^{\circ}$ indicating volume or multiple scattering, and $90^{\circ}$ indicating double bounce scattering. In Fig. 4(c), it is observed that the water body has the lowest $\alpha$ values of less than $45^{\circ}$, forest and aquatic vegetation have intermediate $\alpha$ values of around $60^{\circ}$, and urban area and bare land have the highest $\alpha$ values of close to $90^{\circ}$. Therefore, except for the water body, the other classes are difficult to distinguish by the $\alpha$ value.

The $H-\alpha$ plane maps of the six land cover classes are plotted in Fig. 5. It can be seen that if scatter plots of different classes are placed in the same $H-\alpha$ plane, then the overlap area will be very large, which indicates that the $H-\alpha$ decomposition for dual polarization (VV-VH) has low ability to discriminate the scattering mechanism of the objects.

\subsubsection{Feature importance}

A DT method was used to predict the importance of different features in the classification process. The idea of the method is to evaluate how much each feature contributes to each tree in the DT, then take the average value, and finally obtain the contribution of each feature.

The importance of a feature in a single DT is defined as the reduction of the weighted impurity (Gini coefficient) of the feature when all non-leaf nodes are split. The greater the reduction, the more important the feature is. In the process of tree construction, a Gini coefficient is calculated for each feature, the importance of which is the normalized value of the reduction in the Gini coefficient. The calculation method is as follows:

$$
\text { Feature_Importance }=\frac{N_{t}}{N}\left(\text { Gini }-\frac{N_{t r}}{N_{t}} G_{i n i}-\frac{N_{t l}}{N_{t}} \text { Gini }_{l}\right),
$$

where $N$ is the total number of samples, $N_{t}$ is the number of samples of the current node, $N_{t r}$ is the number of samples of the right sub-node after the current node splitting, Gini ${ }_{r}$ is the Gini

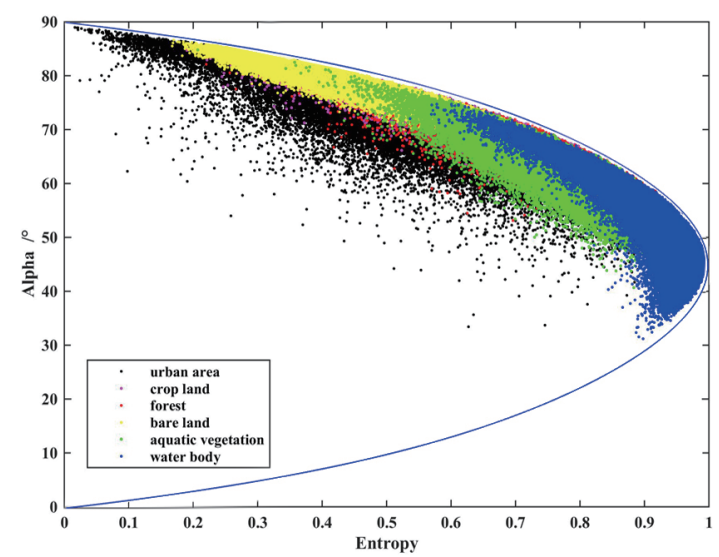

Fig. 5. (Color online) Distribution of scatterers in the dual-polarization $H-\alpha$ plane. 
coefficient of the right sub-node, $N_{t l}$ is the number of samples of the left sub-node after the current node splitting, and $\mathrm{Gini}_{l}$ is the Gini coefficient of the left sub-node.

Figure 6 lists the importance of the six features, which is a measure of the contribution of each feature to the final classification. It can be seen that the backscattering features are generally more important than the polarimetric features. The most important feature is the VV polarization backscattering feature (0.34). Among the polarization features, $\alpha$ is the most important (0.14), while $H$ and $A$ have importances of less than 0.1 .

\subsection{Accuracy assessment}

The classification results of two cases are shown in Table 2: Case I refers to the Poyang Lake region with three land cover classes and Case II refers to the whole image including six land cover classes. In Case I, the classifiers based on the backscattering features, polarimetric

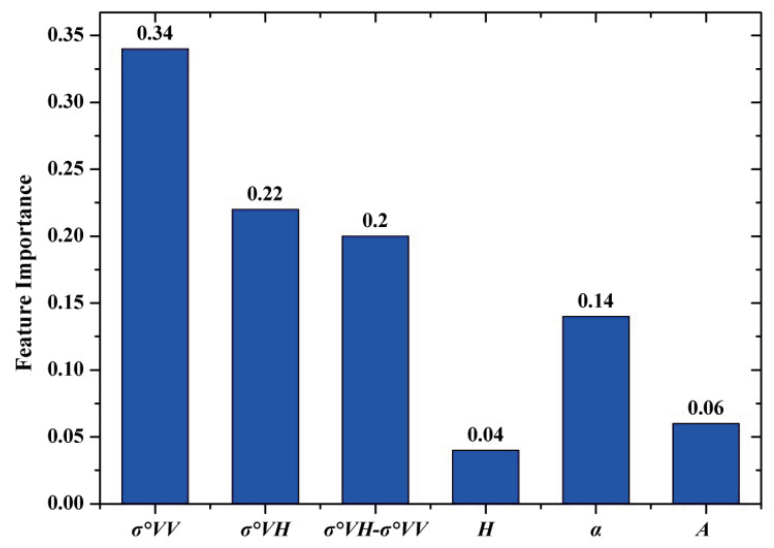

Fig. 6. (Color online) Importance of the six features.

Table 2

Accuracy assessment of land cover classification using different features.

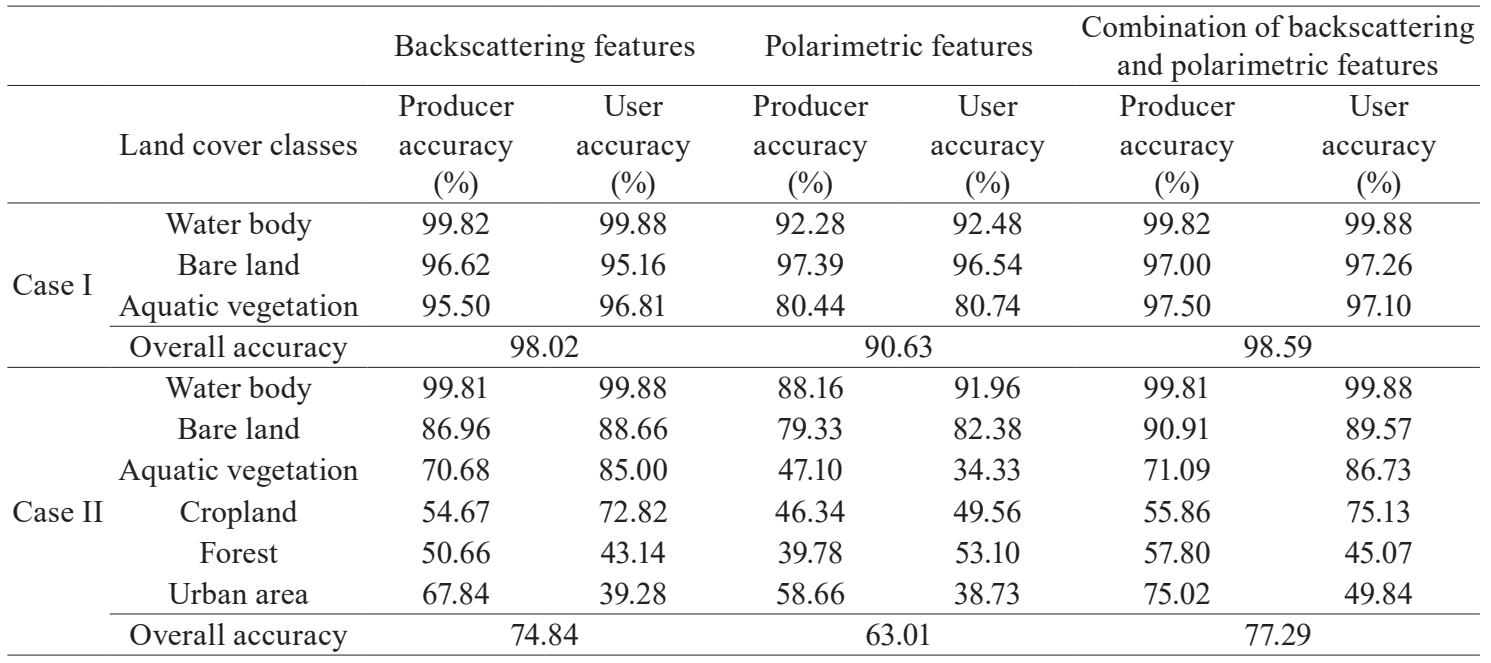




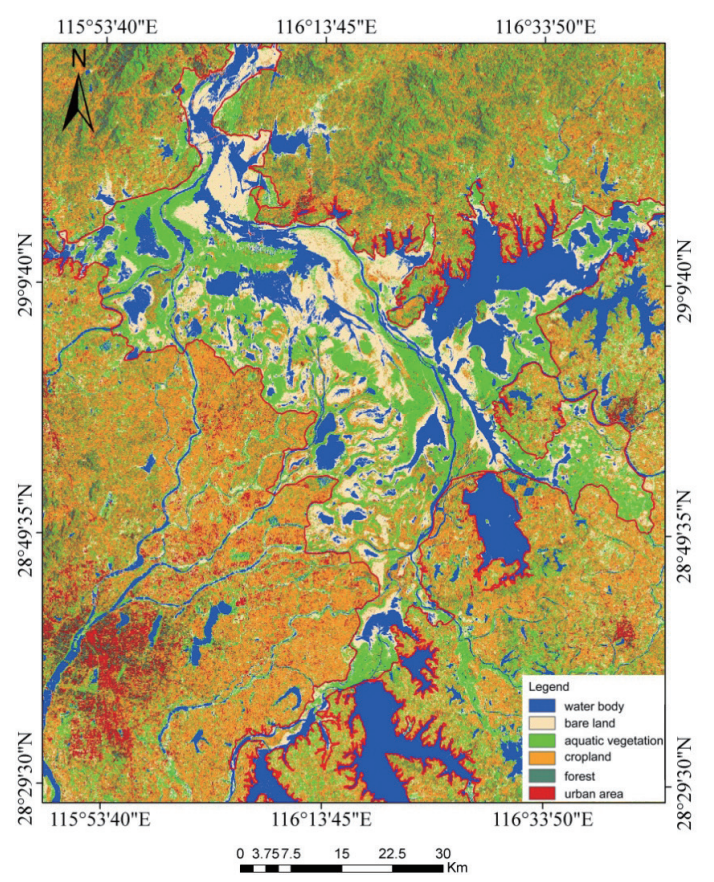

Fig. 7. (Color online) Classification image obtained using backscattering features.

features, and the combination of backscattering and polarimetric features yielded overall accuracy for the three classes of 98.02, 90.63, and 98.59\%, respectively. Meanwhile, in Case II, with the other three land cover classes outside the lake boundary added to the classifiers, the overall accuracy decreased sharply to $74.84,63.01$, and $77.29 \%$, respectively. The main reason for the reduction in the overall classification accuracy is the combination of omission and commission errors, particularly when mixing the three land cover classes in Case I with forest, cropland, and urban area. Moreover, in both cases, it was observed that higher accuracy was achieved by using backscattering features alone than by using polarimetric features alone, and that the classification accuracy of backscattering features was not markedly improved when polarimetric features were added. The reason for the low accuracy of polarimetric feature classification was mainly related to its polarization mode $(\mathrm{VV}-\mathrm{VH})$, which was also consistent with the finding of Ji and Wu that HV-VV SARs can only partially extract low-, medium-, and high-entropy scattering mechanisms due to the lack of co-polarization. ${ }^{(26)}$ Therefore, after comprehensive consideration of the classification accuracy and the polarimetric analysis, it was concluded that the backscattering coefficients were the most appropriate Sentinel-1 features for Poyang Lake wetland classification, as shown in Fig. 7. It can be observed by visually comparing the classified image with the image of the study area (Fig. 1) that most of the classes have been identified with acceptable accuracy. In the Poyang Lake area, the water body, bare land, and aquatic vegetation can be well classified. Beyond the lake, cropland is widely distributed and easily misclassified as forest. 


\subsection{Comparison of different machine learning algorithms}

The artificial neural network (ANN), adaptive boost (AdaBoost), naïve Bayes (NB), and random forest (RF) algorithms were tested while adopting the backscattering feature combination. Specifically, the ANN adopted the back-propagation multilayer perceptron algorithm and established a neural network with four layers. The input layer had three input units, the middle layer had two hidden layers, each hidden layer had six units, and the output layer had one unit. AdaBoost is a typical boosting algorithm that trains weak classifiers in a serial manner on the same training sample set. After each training, it reduces the weight of the correct samples in the training sample set according to the classification results, increases the weight of the incorrect samples, adjusts the weight of a classifier with a small classification error rate, and reduces the classification error. In this study, the number of weak classifiers was set to 10. The NB algorithm is relatively simple and mainly based on the use of probability statistics to discriminate and classify; we used the Gaussian NB algorithm in this study. The number of subclassifiers of the RF classifier was set to 10 and the maximum depth of the sub-DT was set to 10 .

The training and testing accuracies of the different classification methods are shown in Fig. 8. Note that the training accuracy was obtained from the training samples used to determine the parameters of the classifier. The test accuracy was calculated using the test samples, that is, the test samples were predicted using the trained classifier and compared with the known labels to calculate the test accuracy. The results show that the training accuracy and testing accuracy of the different classification methods are very close. Among the four classifiers selected, RF has the highest classification accuracy, followed by ANN and NB, and AdaBoost has the lowest accuracy. The classification results obtained by the four classifiers are shown in Fig. 9. It can be seen that the results of ANN, RF, and NB are very close, but AdaBoost classified farmland as bare land.

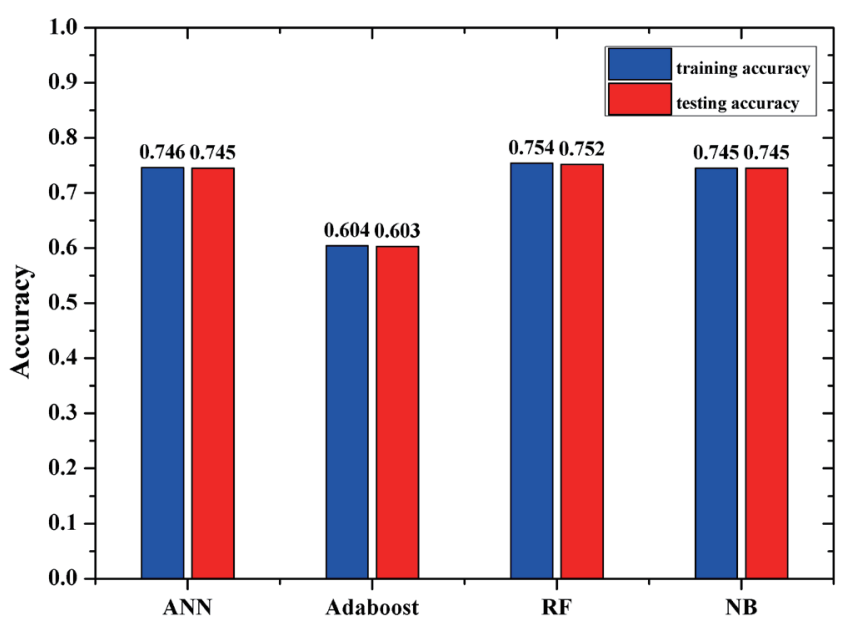

Fig. 8. (Color online) Comparison of classification accuracies. 


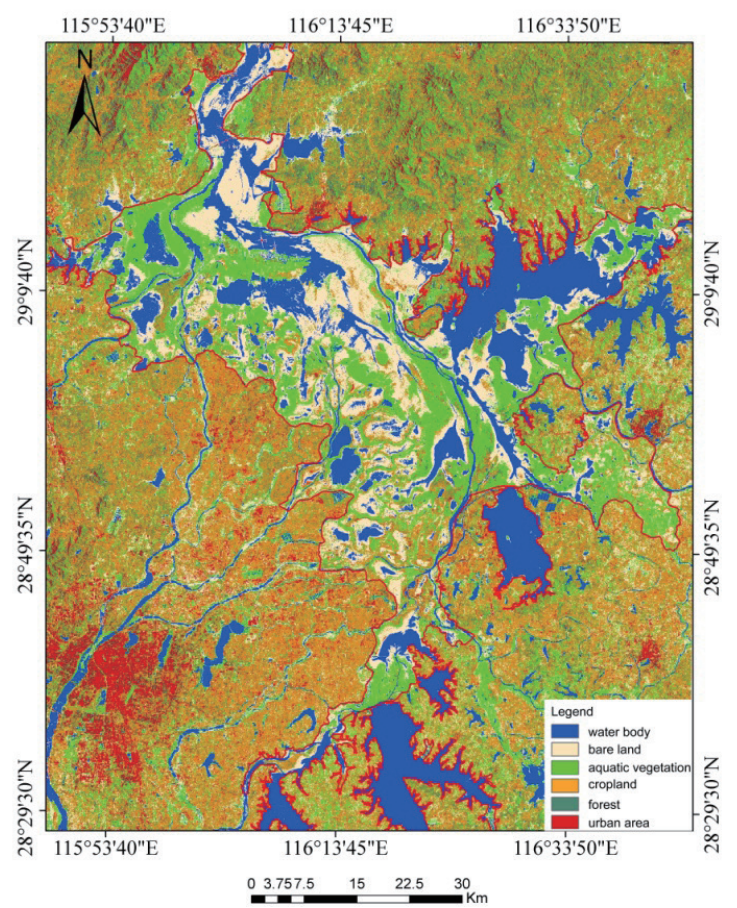

(a)

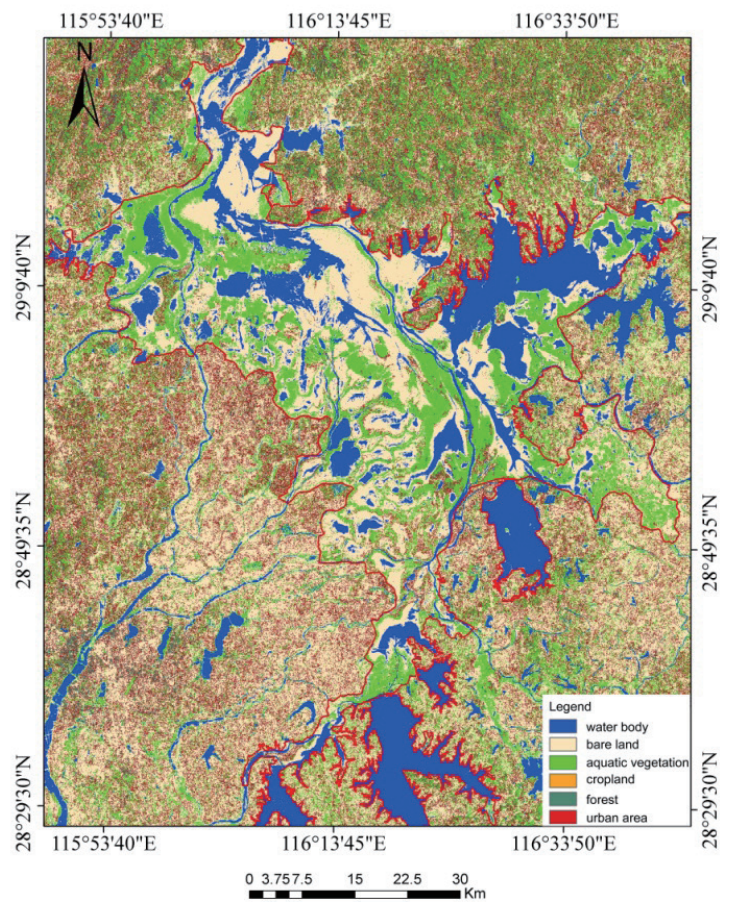

(c)

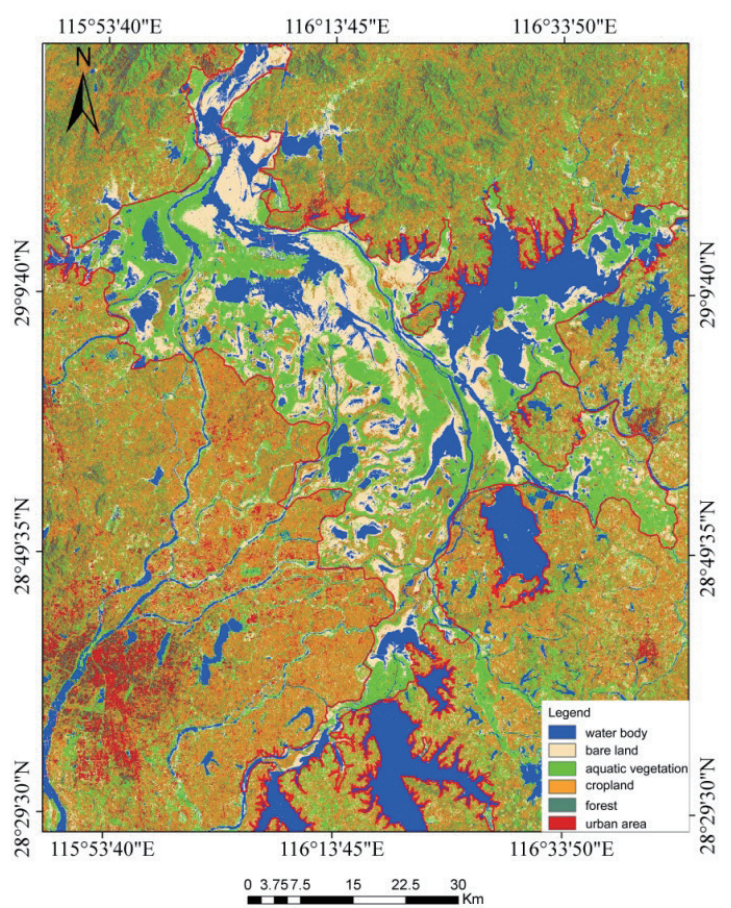

(b)

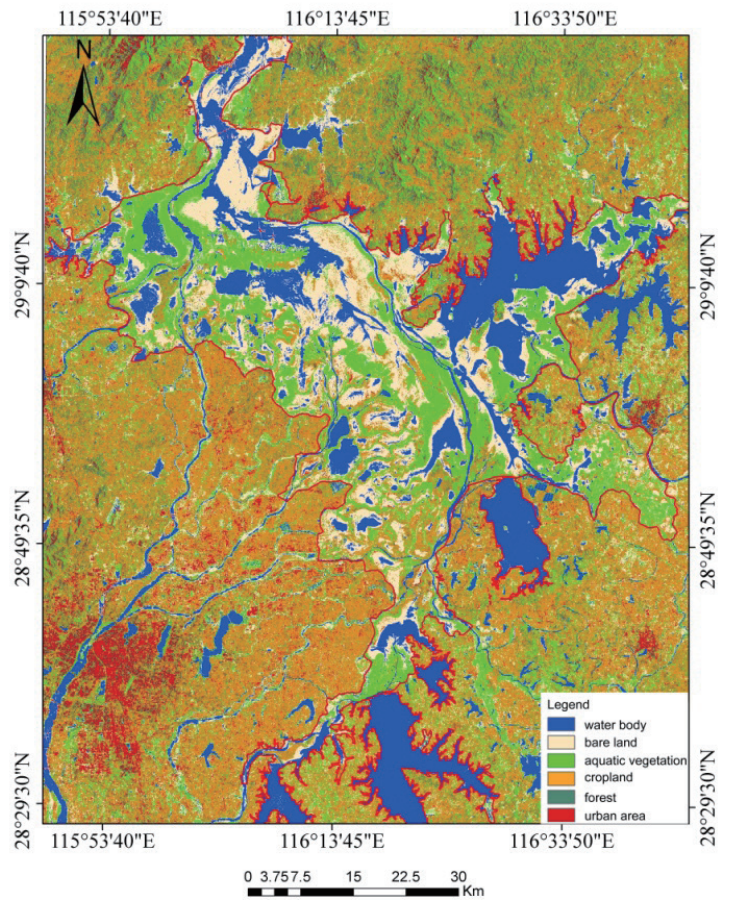

(d)

Fig. 9. (Color online) Classification results of four classifiers. (a) ANN, (b) RF, (c) AdaBoost, and (d) NB. 


\section{Conclusions}

We used single-temporal Sentinel-1 dual-polarized SAR data to classify three broad land cover classes of the Poyang Lake region and surrounding area. The backscattering and polarimetric features of different land covers were analyzed and the classification based on three feature combinations was explored by the DT method. The classification results of the Poyang Lake region gave overall accuracies of over $90 \%$ for the three combinations, indicating the benefits of using dual-polarized SAR data and the possibility of using freely available images instead of purchased images. Additionally, the results indicated the effectiveness of different feature combinations with increasing accuracy in the following order: polarimetric features < backscattering features $<$ combination of polarimetric and backscattering features, showing that for the VV-VH polarization, the backscattering features appear to be the optimum features for wetland classification, whereas dual-polarized SAR has poor performance in extracting scattering mechanisms. However, the results are only for one study area under one polarization mode and more research is needed to obtain more robust conclusions.

Compared with single polarization, dual-polarized SAR provides more information of ground object scattering. How to make full use of this information to obtain better classification is a challenging subject in the field, for which more work remains to be done.

\section{Acknowledgments}

This work was financially supported by the National Natural Science Foundation of China (Grant No. 41971320), Beijing Natural Science Foundation (Grant No. 8162028), and the National Key Research and Development Program of China (Grant No. 2019YFE0126400). The authors would like to thank ESA for the free data and software support.

\section{References}

1 F. M. Henderson and A. J. Lewis: Int. J. Remote Sens. 29 (2008) 5809. https://doi.org/10.1080/01431160801958405

2 W. Ji, X. Xu, and D. Murambadoro: Int. J. Remote Sens. 36 (2015) 1763. https://doi.org/10.1080/01431161.2015.1 $\underline{024895}$

3 M. C. Hansen, R. S. Defries, J. R. G. Townshend, and R. Sohlberg: Int. J. Remote Sens. 21 (2000) 1331. https:// doi.org/10.1080/014311600210209

4 M. W. Lang, E. S. Kasischke, S. D. Prince, and K. W. Pittman: Remote Sens. Environ. 112 (2008) 4120. https:// doi.org/10.1016/j.rse.2007.08.026

5 Y. Yamagata and Y. Yasuoka: Proc. 1993 Geoscience and Remote Sensing Symp. IGARSS '93. Better Understanding of Earth Environment (IEEE, 1993) 1614-1616. https://doi.org/10.1109/IGARSS.1993.322073

6 J. N. R. Rio and D. F. Lozano-García: Remote Sens. Environ. 73 (2000) 143. https://doi.org/10.1016/S00344257(00)00089-4

7 S. Arzandeh and J. Wang: Can. J. Remote Sens. 28 (2002) 653. https://doi.org/10.5589/m02-061

8 J. Li and W. Chen: Int. J. Remote Sens. 26 (2005) 5051. https://doi.org/10.1080/01431160500166516

9 J. Liao, G. Shen, and L. Dong: J. Appl. Remote Sens. 7 (2013) 073579. https://doi.org/10.1117/1.JRS.7.073579

10 J. Whitcomb, M. Moghaddam, K. Mcdonald, J. Kellndorfer, and E. Podest: Can. J. Remote Sens. 35 (2009) 54. https://doi.org/10.5589/m08-080

11 A. Lönnqvist, Y. Rauste, H. Ahola, M. Molinier, and T. Häme: Proc. 2006 Forests and Remote Sensing: Methods and Operational TOOLS (2006).

12 R. Touzi: IEEE Trans. Geosci. Remote Sens. 45 (2006) 73. https://doi.org/10.1109/TGRS.2006.886176

13 P. Patel, H. S. Srivastava, and R. R. Navalgund: Curr. Sci. 97 (2009) 529. https://www.jstor.org/stable/24111881 
14 E. Touzi, A. Deschamps, A. M. Demers, and G. Rother: Proc. 4th Int. Workshop Science and Applications of SAR Polarimetry and Polarimetric Interferometry-PolInSAR 2009 (2009) 668.

15 J. Liao and Q. Wang: Proc. 6th Int. Symp. Digital Earth: Data Processing and Applications 7841 (2010) $78411 B$. https://doi.org/10.1117/12.873258

16 Y. Chen, X. He, and J. Wang: Arabian J. Geosci. 8 (2015) 10203. https://doi.org/10.1007/s12517-015-1940-2

17 A. Schmitt and B. Brisco: Water 5 (2013) 1036. https://doi.org/10.3390/w5031036

18 T. Ullmann, A. Schmitt, and T. Jagdhuber: Remote Sens. 8 (2016) 1. https://doi.org/10.3390/rs8121027

19 L. White, K. Millard, S. Banks, M. Richardson, J. Pasher, and J. Duffe: Remote Sens. 9 (2017) 573. https://doi. org $/ 10.3390 /$ rs 9060573

20 S. Adeli, B. Salehi, M. Mahdianpari, L. J. Quackenbush, B. Brisco, H. Tamiminia, and S. Shaw: Remote Sens. 12 (2020) 2190. https://doi.org/10.3390/rs12142190

21 R. Torres, P. Snoeij, D. Geudtner, D. Bibby, M. Davidson, E. Attema, P. Potin, B. Rommen, N. Floury, M. Brown, I. N. Traver, P. Deghaye, B. Duesmann, B. Rosich, N. Miranda, C. Bruno, M. L'Abbate, R. Croci, A. Pietropaolo, M. Huchler, and F. Rostan: Remote Sens. Environ. 120 (2012) 9. https://doi.org/10.1016/j. rse.2011.05.028

22 J. S. Lee and E. Pottier. Polarimetric Radar Imaging: from Basic to Applications [M] (Taylor \& Francis Group, 2009) pp. 229-239.

23 F. Pedregosa, G. Varoquaux, A. Gramfort, V. Michel, B. Thirion, O. Grisel, M. Blondel, P. Prettenhofer, R. Weiss, V. Dubourg, J. Vanderplas, A. Passos, D. Cournapeau, M. Brucher, M. Perrot, and E. Duchesnay: J. Mach. Learning Res. 12 (2011) 2825

24 M. P. F. Costa: Int. J. Remote Sens. 25 (2004) 1817. https://doi.org/10.1080/0143116031000116985

25 B. Marti-Cardona, C. Lopez-Martinez, and J. Dolz-Ripolles: Remote Sens. Environ. 114 (2010) 2802. https:// doi.org/10.1016/j.rse.2010.06.015

26 K. Ji and Y. Wu: Remote Sens. 7 (2015) 7447. https://doi.org/10.3390/rs70607447 